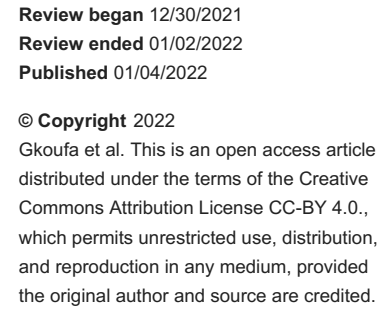

\title{
An Unusual Presentation of Diffuse Large B-Cell Lymphoma
}

\author{
Aikaterini Gkoufa $^{1}$, Vasiliki E. Georgakopoulou ${ }^{2,3}$, Eleftheria Lakiotaki ${ }^{4}$, Evangelos Cholongitas ${ }^{1}$ \\ 1. First Department of Internal Medicine, Laiko General Hospital, School of Medicine, National and Kapodistrian \\ University of Athens, Athens, GRC 2. Department of Pulmonology, Laiko General Hospital, Athens, GRC 3. First \\ Department of Pulmonology, Sismanogleio Hospital, Athens, GRC 4. First Department of Pathology, School of \\ Medicine, National and Kapodistrian University of Athens, Athens, GRC
}

Corresponding author: Vasiliki E. Georgakopoulou, vaso_georgakopoulou@hotmail.com

\begin{abstract}
Hyperlactemia is a rare and potentially fatal complication of hematologic malignancies, as well as an oncological emergency, which requires a fast diagnosis and early therapeutic management, as these interventions may alter disease prognosis. Herein, we present a case of secondary liver-biopsy-confirmed diffuse large B-cell lymphoma (DLBCL), presented with elevated liver enzymes and lactic acidosis, without depicted hepatic lesions, hepatosplenomegaly, or enlarged lymph nodes on computed tomography. This case confirms the poor prognosis of cases with delayed diagnostic intervention and highlights the importance of early clinical suspicion, liver biopsy, and prompt treatment initiation.
\end{abstract}

Categories: Internal Medicine, Oncology, Hematology

Keywords: liver infiltration, diffuse large b-cell lymphoma, liver biopsy, liver lymphoma, lactic acidosis

\section{Introduction}

Diffuse large B-cell lymphoma (DLBCL) is the most common B-cell non-Hodgkin lymphoma (NHL) in adults and secondary involvement of the liver is relatively common in the course of the disease, occurring in 16$46 \%$ of patients [1,2]. Any unusual but urgent manifestation related to DLBCL, such as type B lactic acidosis, hypoglycemia, and renal infiltration of acute liver failure [1,3,4], should raise the suspicion of the clinician to make a prompt diagnosis, especially in cases where rapid treatment commencement may alter disease prognosis. Type B lactic acidosis, occurring under adequate tissue perfusion, as opposed to type A, where tissue hypoxia is present, is a rare and potentially fatal complication of hematologic malignancies [5]. It is also associated with poor prognosis and included in oncological emergencies [5]. The mechanism underlying its pathogenesis may be multifactorial and may portend severe hepatic or renal dysfunction, where lactate metabolism is impaired, thus leading to its accumulation [6]. Other possible mechanisms involve enhanced aerobic glycolytic activity in tumor cells, a process called the "Warburg effect," which is the overexpression of certain glycolytic enzymes [6]. This process may simultaneously cause hypoglycemia or a deficiency of thiamine or riboflavin [7].

There are reports of NHL cases, either presented initially or during disease progression or recurrence, with lactic acidosis with or without hypoglycemia and with or without liver involvement [3,4,6-14]. All these reports indicate a high mortality rate, and any treatment delay may further contribute to devastating outcomes [3,4,6-14]. Herein, we report a patient who presented with fever, elevated liver enzymes, and type $B$ lactic acidosis, diagnosed with DLBCL after a liver biopsy.

\section{Case Presentation}

A 72-year-old Caucasian woman with a past medical history of hypercholesterolemia, treated with pitavastatin, presented to our department due to a two-month history of low-grade fever, weakness, and night sweats. On admission, the patient was febrile and normotensive, while the physical examination was unremarkable. Laboratory analysis showed normochromic, normocytic anemia with elevated C-reactive protein (CRP), erythrocyte sedimentation rate (ESR), lactate dehydrogenase (LDH), and mildly elevated liver enzymes (Table 1 ). 


\section{Cureus}

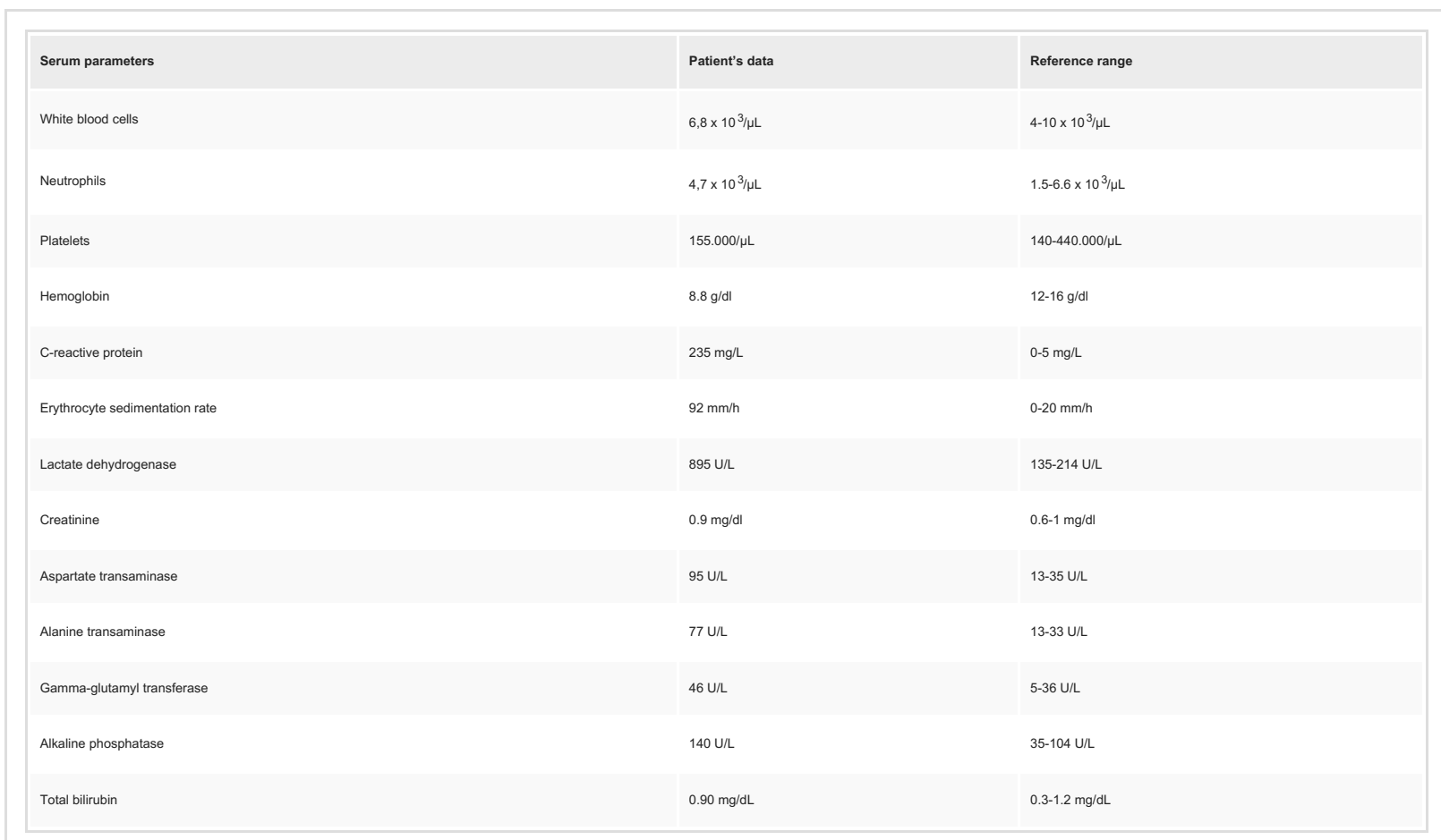

TABLE 1: Laboratory investigations of the patient on admission.

Multiple blood tests, urine cultures, and a purified protein derivative (PPD) test were negative. Viral serology tests, including hepatitis A virus (HAV), hepatitis B virus (HBV), hepatitis C virus (HCV), Epstein-Barr virus (EBV), cytomegalovirus (CMV), and human immunodeficiency virus (HIV), as possible causes for increased liver function tests, were not indicative of a recent or chronic infection. Laboratory studies with antinuclear antibodies (ANA), antimitochondrial antibodies (AMA), anti-liver-kidney microsomal antibodies, and antisoluble liver antigens were negative. A peripheral blood smear was evaluated and did not show any abnormal cells.

Computed tomography (CT) of the brain, chest, and abdomen did not reveal any findings. On the fourth day of hospitalization, and as the patient continued to be febrile, thus meeting the criteria for a fever of unknown origin, a venous blood gas revealed an elevated lactate level ( $8 \mathrm{mmol} / \mathrm{L}), \mathrm{pH}(7.39)$, and bicarbonate (HCO3) $(20.3 \mathrm{mmol} / \mathrm{L})$. Sepsis, cardiogenic shock, and hypoperfusion were excluded due to the patient's normal blood pressure and newly obtained sterile blood and urine culture. Further evaluation with gastrointestinal endoscopies was unremarkable. Due to the marked rise of liver enzymes, lactate level, and LDH, we decided to perform a liver and bone marrow biopsy. Bone marrow aspiration was not diagnostic. The bone marrow biopsy results were delayed, while liver biopsy diagnosed DLBCL (Figures $1 \mathrm{~A}, 1 \mathrm{~B}$ ). A few days later, and while the patient continued to deteriorate with a lactate level of $10.6 \mathrm{mmol} / \mathrm{L}$ and $\mathrm{pH}$ of 7.33 , a bone marrow biopsy confirmed DLBCL (Figures $1 C, 1 D$ ). Soon after diagnosis, and before any treatment initiation, the patient died due to severe respiratory failure arising from hospital-acquired pneumonia after a prolonged hospital course. 


\section{Cureus}
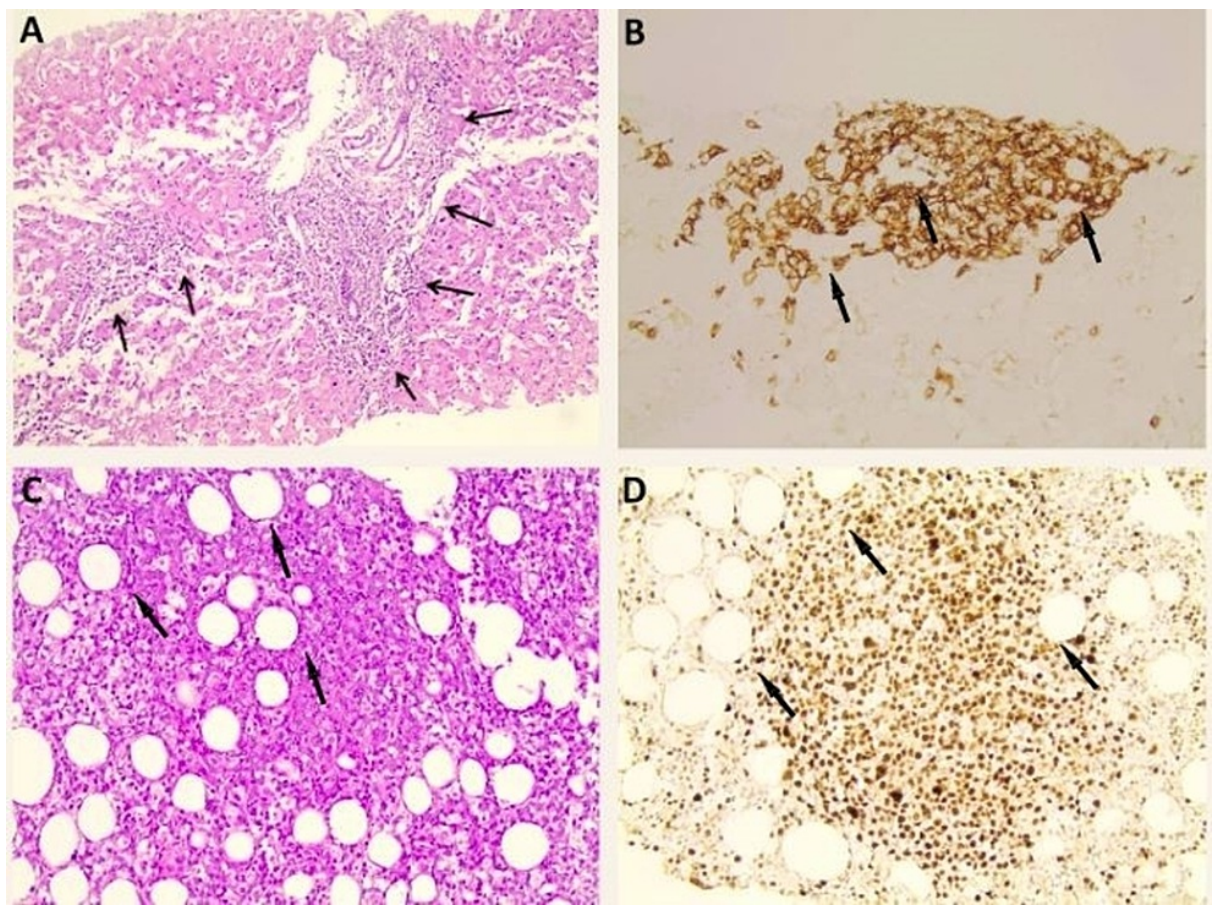

\section{FIGURE 1: Pathology findings from liver and bone marrow biopsy.}

A-B: Pathology findings from liver biopsy. Arrows show extended portal tracts due to diffuse infiltration by largesized lymphoid cells, with a minimal sinusoidal pattern. The lymphoid cells were positive for the B cell marker CD20 and negative for the T cell marker CD3. C-D: Pathology findings from bone marrow biopsy. Arrows show diffuse extensive infiltration by large lymphoid cells that were positive for the markers CD20, CD5, MUM1, c-myc, $\mathrm{BCl}-2$, and $\mathrm{BCl}-6$, and negative for $\mathrm{CD} 3, \mathrm{CD} 10, \mathrm{CD} 30$, and cyclin D1, with a very high proliferation index Ki67 $(90 \%)$, consistent with CD5+ DLBCL of non-germinal center (non-GC) subtype.

DLBCL: diffuse large B-cell lymphoma; MUM1: multiple myeloma oncogene-1; Bcl-2: B-cell lymphoma 2; Bcl-6: Bcell lymphoma 6.

\section{Discussion}

Liver biopsy is the mainstay in the diagnosis of primary hepatic lymphoma, while secondary NHLs are usually diagnosed after a lymph node biopsy, or in rarer cases, after bone marrow aspiration and biopsy [15]. In the literature, there are only three cases of secondary hepatic NHL, presented with lactic acidosis and diagnosed with liver biopsy $[10,16]$. Table 2 summarizes the cases of lymphoma presented with lactic acidosis and diagnosed with liver biopsy.

\begin{tabular}{|c|c|c|c|c|}
\hline Case & Age/sex & Imaging findings & Diagnosis based on liver biopsy & Outcome \\
\hline Kestler et al. (2010) [10] & 40 years $/$ male & Hepatomegaly with multiple lesions, Iymphadenopathy & Burkitt lymphoma & Death seven months after diagnosis \\
\hline Kestler et al. (2010) $[10]$ & 48 years/male & Hepatomegaly with multiple lesions & DLBCL & Death soon after presentation \\
\hline Keller et al. (2010) [16] & 53 years/male & Hepatomegaly, splenomegaly, Iymphadenopathy & DLBCL & Treatment response \\
\hline Our case & 72 years/fiemale & Normal & DLBCL & Death at initial presentation \\
\hline
\end{tabular}

TABLE 2: Cases of lymphoma presented with lactic acidosis and diagnosed with liver biopsy.

DLBCL: diffuse large B-cell lymphoma.

Kestler et al. reported a patient with a recent diagnosis of HIV infection, who presented with night sweats, dysphagia, and postprandial abdominal pain and elevated LDH, lactate level, and liver enzymes [10]. His abdominal CT revealed hepatomegaly with multiple lesions and associated bulky adenopathy of the gastroesophageal junction, while the liver biopsy showed infiltration of DLBCL [10]. In the same report, Kestler et al. reported a second patient with a prior history of HIV infection, who presented with dyspnea, abdominal discomfort, and hyperlactatemia [10]. Due to significant hepatomegaly and elevated liver 
enzymes, the performance of liver biopsy was decided; however, the patient died after multi-organ system failure and a post-mortem liver biopsy revealed DLBCL [10]. Keller et al. reported a patient with fever, nausea, abdominal discomfort, diarrhea, elevated lactate level, hypoglycemia, and increased liver function tests, with hepatomegaly, splenomegaly, and abdominal lymphadenopathy. The patient's liver biopsy demonstrated a DLBCL [16]. All patients had image confirmation of liver involvement [16].

To the best of our knowledge, this is the first reported case of DLBCL presented with laboratory findings of lactic acidosis and elevated liver enzymes, and diagnosed with liver biopsy, without depicting hepatic lesions, hepatomegaly, or enlarged lymph nodes on abdominal CT. Imaging features of secondary hepatic lymphoma may range from diffuse infiltration to multifocal homogenous lesions or miliary patterns, with extrahepatic involvement (splenic lesions, lymphadenopathy, and bone marrow infiltration) [17]. Despite relatively common liver involvement in NHLs, in rare cases, liver and bone marrow may be the only sites of the disease, thus complicating diagnosis, especially in cases where hepatomegaly or focal hepatic lesions are absent [18]. In such cases, and as liver involvement is considered as evidence of advanced disease and the diffuse infiltration of the liver has no specific imaging features, any abnormality of liver enzymes should be promptly evaluated and a liver biopsy should be obtained as early as possible [18]. In our patient, fluorodeoxyglucose positron emission tomography-computed tomography (FDG-PET/CT) was potentially helpful for biopsy guidance; however, this diagnostic test was not available in our hospital and the patient's clinical deterioration during hospitalization was a deterrent factor for her referral. Concerning lactic acidosis, after the exclusion of hypoperfusion, it was finally attributed both to liver involvement and the metabolic effects of the lymphoma. Although bicarbonate and thiamine administration, as well as hemodialysis, have been used for the management of type B lactic acidosis, the only effective management focuses on the treatment of underlying malignancy and identification $[12,13,19]$. The inciting etiology constitutes the main challenge for clinicians [19].

\section{Conclusions}

Type B lactic acidosis, an uncommon and challenging presenting feature of hematologic malignancies, is associated with poor prognosis and highlights that timely detection of the underlying disease is crucial for patients' improvement and survival. Unexplained lactic acidosis in a patient with increased liver function tests, even in cases without focal hepatic lesions or hepatomegaly, should raise strong suspicions and guide the clinician in performing a liver biopsy without delay. Most hematologists are quite familiar with this manifestation in patients with an already known hematologic malignancy; however, an undiagnosed patient, hospitalized in an internal medicine department, requires the vigilance of experienced physicians and clinicians to provide an efficient evaluation, quick diagnosis, and early therapeutic management.

\section{Additional Information}

\section{Disclosures}

Human subjects: Consent was obtained or waived by all participants in this study. Conflicts of interest: In compliance with the ICMJE uniform disclosure form, all authors declare the following: Payment/services info: All authors have declared that no financial support was received from any organization for the submitted work. Financial relationships: All authors have declared that they have no financial relationships at present or within the previous three years with any organizations that might have an interest in the submitted work. Other relationships: All authors have declared that there are no other relationships or activities that could appear to have influenced the submitted work.

\section{References}

1. Hafner A, Eaton DB: Acute liver failure with severe lactic acidosis secondary to infiltrative diffuse large Bcell lymphoma: an imaging-negative presentation. Cureus. 2020, 12:e10110. 10.7759/cureus.10110

2. Peng Y, Qing AC, Cai J, Yue C, French SW, Qing X: Lymphoma of the liver: clinicopathological features of 19 patients. Exp Mol Pathol. 2016, 100:276-80. 10.1016/j.yexmp.2016.02.001

3. Glasheen JJ, Sorensen MD: Burkitt's lymphoma presenting with lactic acidosis and hypoglycemia - a case presentation. Leuk Lymphoma. 2005, 46:281-3. 10.1080/10428190400016723

4. Tanios G, Aranguren IM, Goldstein JS, Patel CB: Diffuse large B-cell lymphoma: a metabolic disorder?. Am J Case Rep. 2013, 14:518-25. 10.12659/ajcr.889580

5. De Raes EA, Benoit DD, Depuydt PO, et al.: Early recognition of malignant lactic acidosis in clinical practice: report on 6 patients with haematological malignancies. Acta Clin Belg. 2012, 67:347-51.

6. Liberti MV, Locasale JW: The Warburg effect: how does it benefit cancer cells? . Trends Biochem Sci. 2016, 41:211-8. 10.1016/i.tibs.2015.12.001

7. Friedenberg AS, Brandoff DE, Schiffman FJ: Type B lactic acidosis as a severe metabolic complication in lymphoma and leukemia: a case series from a single institution and literature review. Medicine (Baltimore). 2007, 86:225-32. 10.1097/MD.0b013e318125759a

8. Mintz U, Sweet DL Jr, Bitran JD, Ultmann JE: Lactic acidosis and diffuse histiocytic lymphoma (DHL). Am J Hematol. 1978, 4:359-65. 10.1002/ajh.2830040407

9. Sillos EM, Shenep JL, Burghen GA, Pui CH, Behm FG, Sandlund JT: Lactic acidosis: a metabolic complication of hematologic malignancies: case report and review of the literature. Cancer. 2001, 92:2237-46. 10.1002/1097-0142(20011101)92:9<2237::AID-CNCR1569>3.0.CO;2-9

10. Kestler MH, Gardner EM, Cohn DL: Hepatic non-Hodgkin's lymphoma with lactic acidosis in HIV-infected 


\section{Cureus}

patients: report of 2 cases. J Int Assoc Physicians AIDS Care (Chic). 2010, 9:301-5.

$10.1177 / 1545109710377803$

11. Kumar A, Raina V: Non-Hodgkins lymphoma with lactic acidosis at presentation: a case report of a rare oncologic emergency. Indian J Med Paediatr Oncol. 2014, 35:83-5. 10.4103/0971-5851.133728

12. Arif H, Zahid S, Kaura A: Persistent lactic acidosis: thinking outside the box. Cureus. 2018, 10:e2561. 10.7759/cureus.2561

13. He Y, Ong J, Ong S: Refractory lactic acidosis and an approach to its management - a case report . J Crit Care Med (Targu Mures). 2019, 5:60-5. 10.2478/jccm-2019-0010

14. Masood U, Sharma A, Nijjar S, Sitaraman K: B-cell lymphoma, thiamine deficiency, and lactic acidosis. Proc (Bayl Univ Med Cent). 2017, 30:69-70. 10.1080/08998280.2017.11929534

15. Cozzolino I, Rocco M, Villani G, Picardi M: Lymph node fine-needle cytology of non-Hodgkin lymphoma: diagnosis and classification by flow cytometry. Acta Cytol. 2016, 60:302-14. 10.1159/000448389

16. Keller BC, Nussensveig D, Dowell JE: Diffuse large B-cell lymphoma in a hepatitis C virus-infected patient presenting with lactic acidosis and hypoglycemia. Am J Med Sci. 2010, 339:202-4.

10.1097/MAJ.0b013e3181c3fdeb

17. Alves AMA, Torres US, Velloni FG, Ribeiro BJ, Tiferes DA, D'Ippolito G: The many faces of primary and secondary hepatic lymphoma: imaging manifestations and diagnostic approach. Radiol Bras. 2019, 52:32530. 10.1590/0100-3984.2018.0013

18. Rajesh S, Bansal K, Sureka B, Patidar Y, Bihari C, Arora A: The imaging conundrum of hepatic lymphoma revisited. Insights Imaging. 2015, 6:679-92. 10.1007/s13244-015-0437-6

19. Wahab A, Kesari K, J Smith S, Liu Y, Barta SK: Type B lactic acidosis, an uncommon paraneoplastic syndrome. Cancer Biol Ther. 2018, 19:101-4. 10.1080/15384047.2017.1394550 